\title{
IC_AOMDV: Um protocolo de roteamento capaz de melhorar a conectividade e o desempenho das VANETs
}

\author{
Danilo R. de Assis, Joilson Alves Junior, Emilio C. G. Wille
}

\begin{abstract}
Resumo-As Redes Ad Hoc Veiculares (VANETs) fazem parte dos Sistemas Inteligentes de Transporte (ITS) e têm por objetivo prover a comunicação entre veículos. Nas VANETs, os principais desafios da comunicação estão relacionados à problemas de conectividade (devido ao reduzido tempo de duração das rotas). Este trabalho propõe o Improved Connectivity Ad Hoc On-Demand Multipath Distance Vector (IC_AOMDV), que utiliza fatores relacionados à conectividade (densidade e sentido dos deslocamentos dos nós) para a construção das rotas. As simulações demonstram que o IC_AOMDV é capaz de aumentar o tempo de duração das rotas e a taxa de entrega dos pacotes, enquanto diminui o índice de quebra de rotas.
\end{abstract}

Palavras-Chave-VANETs, V2V, protocolos de roteamento, conectividade, tempo de duração de rota.

Abstract-Vehicular Ad Hoc Networks (VANETs) are part of Intelligent Transportation Systems (ITS) and aim to provide communication between vehicles. In VANETs, the main communication challenges are related to connectivity problems due to short route duration times. This paper proposes the Improved Connectivity Ad Hoc On-Demand Multipath Distance Vector (IC_AOMDV), a routing protocol capable of improving the network performance considering density and movement direction of the nodes.The simulations show that IC_AOMDV is able to increase the route duration time and the packet delivery rate, while reducing route interruption index.

Keywords-VANETs, V2V, routing protocols, connectivity, route duration time.

\section{INTRODUÇÃO}

As redes Ad Hoc veiculares (VANETs) são redes onde a comunicação é estabelecida entre veículos (nós). Quando esta comunicação é realizada de forma dinâmica, sem o intermédio de uma estrutura centralizadora, ela é conhecida como V2V (Vehicule-to-Vehicle). As VANETs fazem parte dos Sistemas Inteligentes de Transporte (ITS) e devem ser capazes de prover segurança e entretenimento aos motoristas e passageiros [1], [2].

As redes $\mathrm{V} 2 \mathrm{~V}$ se caracterizam por serem auto organizadas e auto gerenciadas e com controle descentralizado. Essas características permitem que elas possam ser construídas em qualquer lugar, pois não existe um elemento centralizador, sendo consideradas de baixo custo financeiro [3], [4]. Os nós de tais redes se comunicam uns com os outros por meio de sinais de radiofrequência. Como o alcance dos sinais de rádio é limitado, cada nó só pode se comunicar diretamente com veículos que estiverem dentro do raio de transmissão de seus sinais [1]. Entretanto, pode existir a necessidade de um veículo transmitir informações para outros veículos que estão além do seu raio de transmissão. Para isso, os veículos devem cooperar entre si, agindo como roteadores (descobrindo rotas e

D. R. Assis, J. Alves Jr e E. C. G. Wille, Universidade Tecnológica Federal do Paraná (UTFPR), Av. Sete de Setembro 3165, 80230-901, Curitiba (PR), Brazil. E-mail: daniloassis@utfpr.edu.br, joilson@utfpr.edu.br, ewille@utfpr.edu.br. repassando as informações da origem até o destino) [5], [6]. O envio/recebimento das informações ocorre somente quando um veículo de origem possui uma rota para um veículo de destino. Como as redes V2V não possuem controle centralizado, o seu desempenho é totalmente dependente da existência de rotas e do tempo em que as mesmas permanecem estabelecidas (enquanto uma rota permanecer estabelecida, a origem e o destino são capazes de transmitir dados) [5], [6].

Nas VANETs, os principais desafios da comunicação estão relacionados a problemas de conectividade entre os nós (devido ao reduzido tempo de duração das rotas) e ao baixo desempenho dos protocolos de roteamento clássicos. A literatura acadêmica tem relatado estes problemas e proposto diversas soluções, utilizando uma infraestrutura fixa em conjunto com protocolos de roteamento [7], [8], [2]. Entretanto, o uso de uma infraestrutura fixa pode elevar os custos, o que as tornam impraticáveis [4]. Por esta razão, devese considerar sempre que possível o uso de redes V2V.

Nas redes V2V, a conectividade é totalmente dependente do tempo de duração das rotas e de fatores como: a velocidade, a densidade, o sentido dos deslocamentos e o raio de transmissão dos veículos. Conhecer e avaliar este tempo e quais destes fatores apresentam maior influência, pode proporcionar o desenvolvimento de protocolos e aplicações mais adequados para maximizar a conectividade da rede, assim como contribuir para o desenvolvimento de uma infraestrutura de comunicação veicular mais eficiente [1], [6].

A maioria dos trabalhos sobre o tempo de duração das rotas são realizadas por meio de estudos analíticos [9], [10], [11]. Tais estudos levam em consideração os fatores influentes mas não consideram a estrutura viária (ruas, quadras, cruzamentos e semáforos) e o fluxo de veículos (congestionamentos). Assim, alguns de seus resultados podem não representar fielmente a realidade dos movimentos veiculares. O estudo em [6] apresentou uma análise sobre o tempo de duração das rotas considerando um conjunto de fatores, com o objetivo de revelar quais destes fatores mais contribuem para o aumento da conectividade da rede em ambientes reais. Os autores evidenciaram que o tempo de duração de rotas não varia significativamente em função da velocidade dos veículos quando esta é limitada pelas restrições de mobilidade de um ambiente urbano. Além disso, o estudo demonstrou que o tempo de duração das rotas aumenta à medida que a rede fica mais densa e quando os veículos trafegam no mesmo sentido, pois a probabilidade de interrupção dos enlaces de comunicação torna-se menor.

Portanto, este trabalho tem por objetivo propor a construção de um novo protocolo de roteamento, chamado de IC_AOMDV, que leva em conta os fatores que mais influenciam a conectividade da rede. O IC_AOMDV pode descobrir múltiplas rotas entre a origem e o destino e utilizar como primeira opção aquela que obtiver o melhor valor para 
uma métrica apropriada, sendo que as outras somente são utilizadas caso a primeira apresente falha. Este novo protocolo (independentemente de infraestrutura externa, e.g., um sistema GPS) é capaz de manter a rede operando pelo maior tempo possível. A construção do IC_AOMDV é baseada do protocolo clássico AOMDV [12].

O restante deste trabalho está organizado da seguinte forma: a Seção II exibe o protocolo proposto; a Seção III apresenta a avaliação de desempenho do protocolo IC_AOMDV; a Seção IV mostra os resultados de simulação e, a Seção V apresenta a conclusão.

\section{O PROTOCOLO PROPOSTO}

O Improved Connectivity Ad Hoc On-Demand Multipath Distance Vector (IC_AOMDV) é uma extensão do protocolo de roteamento AOMDV. Ele foi desenvolvido para considerar os fatores influentes densidade e sentido dos deslocamentos dos veículos no processo de descoberta de rotas, e assim selecionar caminhos com maior conectividade, favorecendo o desempenho do sistema. O ponto chave da proposta se concentra no processo de descoberta de rotas do protocolo [13].

O AOMDV é um protocolo reativo, isto é, somente descobre rotas quando é realmente necessário. O processo de descoberta de rotas se inicia quando a origem envia uma mensagem Route Request (RREQ), em broadcast, para todos seus vizinhos. Os nós vizinhos recebem cópias desta mensagem e verificam na tabela de roteamento se existe uma ou mais rotas válidas até o destino. Caso exista, o nó então cria a mensagem Route Reply (RREP) e envia para a origem por meio do caminho reverso criado pelas mensagens de RREQ (nós intermediários que recebem cópias das mensagens de RREQ, criam caminhos reversos, livres de laços, pelos quais a mensagem de RREP retorna para a origem). Caso não exista uma rota válida, o nó então encaminha a RREQ para os seus vizinhos até que o destino seja encontrado [12].

\section{A. A seleção da melhor rota}

A escolha das rotas por onde os pacotes são enviados é importante e pode determinar o tempo de duração das rotas [10]. Nos protocolos de roteamento clássicos, esta escolha é geralmente feita por meio do menor número de saltos. Entretanto, a rota mais curta nem sempre é a rota mais conectada [12]. Dessa forma, é necessário utilizar outros fatores que combinados com o número de saltos possam determinar o caminho mais conectado. O IC_AOMDV adota uma nova métrica, a qual utiliza os fatores influentes (sentido dos deslocamentos e densidade veicular), combinados com o número de saltos, para identificar as melhores rotas.

Nas redes V2V, é desafiador determinar o sentido dos deslocamentos pois não existe infraestrutura fixa. Uma solução consiste na adoção de uma bússola eletrônica (obtida na prática a partir de um magnetômetro) instalada em cada veículo [14]. Definem-se por nós favoráveis aqueles, pertencentes a uma possível rota entre origem e destino, que não se movimentam em direção oposta à direção do movimento do nó origem. Formalmente, seja $\theta j$ o ângulo formado entre o vetor que indica o movimento do nó $j$ e o vetor que indica o norte magnético. Este ângulo pode ser facilmente obtido com uso do magnetômetro instalado nos nós. Seja $N_{i}$ o conjunto de nós pertencentes à rota $i$. Seja diff $=\left|\theta_{\text {origem }}-\theta_{j}\right|$, a diferença entre os ângulos associados a um nó origem e um nó $j$ qualquer. Seja $I_{j}$ uma função indicadora que assume o valor 1 se $\operatorname{diff} \leq 90^{\circ}$ e zero caso contrário. Assim, o número de nós favoráveis, $N$ dir, para uma dada rota é determinado por:

$$
N \operatorname{dir}=\sum_{j \in N_{i}} I_{j}
$$

A Figura 1(a) apresenta quatro veículos (A, B, C e D) e seus ângulos $\left(\theta_{A}, \theta_{B}, \theta_{C}\right.$ e $\left.\theta_{D}\right)$, durante o processo de descoberta de rotas. O veículo A é a origem e os demais veículos são os possíveis candidatos a nós favoráveis de uma rota qualquer. A seta interna ao veículo aponta o norte magnético. É possível observar que os nós $\mathrm{B}$ e C são considerados nós favoráveis pois o diff é $\leq 90^{\circ}$. Entretanto, o diff entre o ângulo da origem e $\theta_{D}$ não satisfaz a condição e portanto não é considerado um nó favorável.

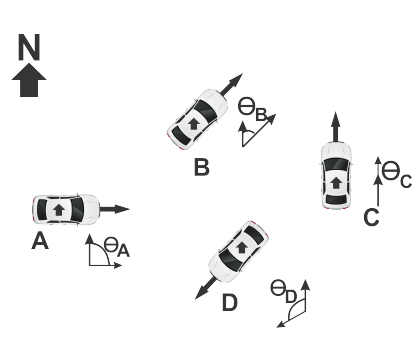

(a) Sentido dos deslocamentos

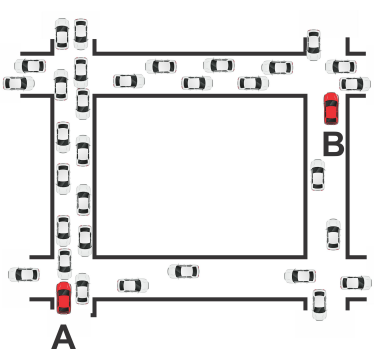

(b) Densidade veicular
Fig. 1. Ilustração gráfica dos fatores influentes

Por outro lado, a densidade veicular pode ser estimada a partir da coleta dos pacotes de controle que um dado nó envia e recebe. Quanto mais pacotes de controle passam pelo nó, mais densa será a região em que ele está localizado [1]. Neste trabalho, considera-se a quantidade de mensagens (pacotes) HELLO que os nós recebem em um dado intervalo de tempo. Esta quantidade de HELLOs (denotada por densidadeequivalente) será, então, proporcional à densidade veicular (nós/área) ao redor de um dado nó na rede.

Seja $N_{\text {hello-T,j }}$ o número de mensagens HELLO recebidas por um nó $j$ em um intervalo de tempo $T$, e seja Nhops o número de saltos por rota. Assim, a densidade-equivalente para uma dada rota é determinada por:

$$
D=\frac{1}{N_{\text {hops }}} \sum_{j \in N_{i}} N_{\text {hello-T,j }}
$$

Neste trabalho, foram realizados estudos com intervalos de coleta de 1,3 e 5 segundos e observou-se que o intervalo de 3 s fornece uma boa aproximação da densidade ao redor de um nó. Ainda assim, o cálculo da densidade veicular não gera uma sobrecarga extra em razão da contagem ser realizada com mensagens de roteamento já existentes no protocolo clássico.

A título de exemplo, a Figura 1(b) apresenta um cenário com uma região onde o tráfego de veículos é mais intenso e outra região com um menor número de veículos. As regiões com grande concentração de veículos recebem mais mensagens HELLO quando comparado a regiões com número reduzido de nós. Desta forma, um caminho mais favorável entre a origem $A$ e o destino $B$, seria aquele que faz uso das vias ao topo e à esquerda na figura.

Com base no exposto, determina-se o valor da métrica $M_{i}$, para cada rota possível $i$ entre origem e destino, conforme:

$$
M_{i}=\frac{\left[N \text { hops }_{i} *\left(N \text { hops }_{i}-N \operatorname{dir}_{i}\right)\right]^{\alpha_{1}}}{D_{i}^{\alpha_{2}}},
$$


onde Nhops é o número de saltos, Ndir é o número de nós favoráveis, $D$ corresponde à densidade-equivalente de cada rota encontrada, e $\alpha_{1}$ e $\alpha_{2}$ são parâmetros definidos pelo usuário. A melhor rota é aquela que minimiza o valor de $M_{i}$.

\section{B. Processo de descoberta de rota}

O processo de descoberta de rotas do IC_AOMDV é similar ao protocolo clássico. Quando um nó necessita enviar uma mensagem para outro nó para o qual ele ainda não conhece a rota, uma mensagem RREQ é enviada em broadcast e mensagens RREP são enviadas em resposta pelo destino ou por um nó intermediário conhecedor da rota até a origem. No entanto, os pacotes de roteamento RREQ e RREP foram modificados no IC_AOMDV para coletar os valores dos fatores necessários (densidade e sentido dos deslocamentos). $\mathrm{Na}$ mensagem RREQ foram incluídos três novos campos, os quais são: ang, fvr e dns. O primeiro campo referese ao ângulo de deslocamento da origem no momento que a mensagem de RREQ é formada. O segundo campo é o número de nós favoráveis ao longo da rota. O último campo corresponde ao somatório da densidade veicular da rota. A Tabela I mostra a estrutura da mensagem modificada do RREQ no IC_AOMDV.

TABELA I

ESTRUTURA DA MENSAGEM DE RREQ DO IC_AOMDV.

\begin{tabular}{|c|c|c|c|c|c|c|c|}
\hline src & dst & dst_seqno & hop_count & liftime & ang & fvr & dns \\
\hline
\end{tabular}

Ao receber uma RREQ, o nó intermediário verifica se a diferença (diff) entre o seu angulo de deslocamento e o angulo da origem, é menor que $90^{\circ}$. Caso seja menor, este nó é um nó favorável e a variável fvr na RREQ é incrementada. Além disso, o nó coleta a informação da densidade veicular ao seu redor, por meio da contagem do número de mensagens HELLO que recebeu de seus vizinhos e incrementando com este total a variável dns na mensagem de RREQ.

Na mensagem de RREP, são incluídos dois novos campos, os quais são: $d n s$ e $f v r$. Ao receber uma mensagem de RREQ, o destino, ou o nó conhecedor da rota para o destino, copia os valores das variáveis $d n s$ e $f v r$ para os campos $d n s$ e $f v r$ da RREP. A Tabela II apresenta a estrutura da mensagem de RREP do protocolo proposto.

\section{TABELA II}

ESTRUTURA DA MENSAGEM DE RREP DO IC_AOMDV.

\begin{tabular}{|c|c|c|c|c|c|c|}
\hline src & dst & dst_seqno & hop_count & liftime & dns & fvr \\
\hline
\end{tabular}

O processo de descoberta de rotas tem por objetivo encontrar múltiplas rotas até o destino. No entanto, este processo também é utilizado para atualizar as informações das tabelas de roteamento dos nós, como por exemplo, manter atualizado o campo hop_count com a quantidade de saltos até o destino. No IC_AOMDV, são incluídos três novos campos na tabela de roteamento, na lista de rotas da origem, os quais são: $d n s, f v r$ e conec. Os campos dns e fvr são atualizados na tabela de roteamento dos nós intermediários com as informações sobre a densidade veicular e o número de veículos favoráveis de cada rota. O campo conec é atualizado, somente no nó de origem, com a métrica de cada rota, de acordo com a Equação 3. Com a nova tabela de roteamento, torna-se possível ordenar as rotas em ordem crescente em função da métrica. A Tabela III mostra um comparativo da estrutura da lista de rotas do AOMDV e do IC_AOMDV.
TABELA III

ESTRUTURA DA LISTA DE ROTAS DO AOMDV E DO IC_AOMDV.

\begin{tabular}{|l|l|}
\hline AOMDV & IC_AOMDV \\
\hline RouteList [(next hop1, \\
$\begin{array}{l}\text { hop count 1),(next hop2, } \\
\text { hop count2),....] }\end{array}$ & $\begin{array}{l}\text { dns1, fvr1, conec1),(next hop2, } \\
\text { hop count2, dns2, fvr2, conec2),.... }\end{array}$ \\
\hline
\end{tabular}

\section{AVAliação DE DESEMPENHO DO IC_AOMDV}

A avaliação do IC_AOMDV consiste em verificar a capacidade do protocolo proposto em melhorar as métricas de desempenho. Para estas análises foi utilizado o simulador de rede Network Simulator (NS-2) [15] e o simulador de mobilidade veicular VanetMobiSim [16].

\section{A. Cenário de simulação}

Neste trabalho é utilizado o cenário de simulação ilustrado na Figura 2, que corresponde uma área retangular de $1000 \mathrm{~m}$ x $1000 \mathrm{~m}$ (subdividida em quatro regiões) com 200 veículos e quadras de $100 \mathrm{~m}$. As regiões 1 e 2 são áreas onde o tráfego é mais denso (com 160 nós) e menos denso (com 40 nós), respectivamente. A região 3 compreende os veículos de origem e a região 4 compreende os veículos de destino. O objetivo deste cenário é demonstrar que o IC_AOMDV escolhe rotas mais conectadas em relação ao protocolo clássico, melhorando o desempenho da rede.

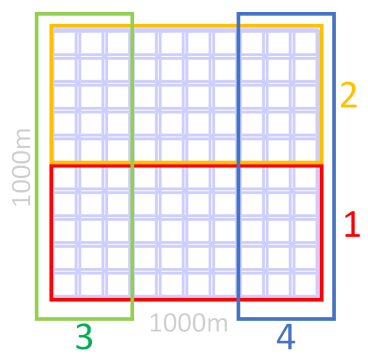

Fig. 2. Cenário de simulação.

\section{B. Métricas para avaliação de desempenho}

O IC_AOMDV foi avaliado considerando as seguintes métricas:

- Taxa de Duração de Rota (TDR): percentagem de tempo onde existe conexão durante o tempo de simulação, i.e., $T D R=(x / t) * 100$, onde $x$ é a média dos tempos de duração de todas as rotas formadas e $t$ é o tempo total de simulação.

- Índice de Quebra de Rota (IQR): quantidade de vezes que ocorreu a quebra de uma rota estabelecida.

- Taxa de Entrega de Pacote (TEP): proporção do número total de pacotes recebidos no destino em relação ao número total de pacotes enviados pela origem.

\section{Configuração das simulações}

Foram utilizados 200 veículos com velocidades que variam entre 10 e $60 \mathrm{~km} / \mathrm{h}$. O raio de transmissão varia entre 150 e $300 \mathrm{~m}$ e o padrão de movimentação dos veículos é gerado pelo VanetMobiSim. O padrão de tráfego consiste de conexões com taxa de bits constante (CBR - 4 mensagens/s), considerando o protocolo de transporte UDP e os protocolos de roteamento AOMDV e IC_AOMDV. O modelo de propagação de rádio 
utilizado é o twoRay ground, enquanto a camada MAC está de acordo com a especificação IEEE 802.11p [17]. São consideradas 5 conexões simultâneas, onde origem e destino são escolhidos aleatoriamente e permanecem desta forma até o fim da simulação. As simulações são realizadas por 600 segundos e todos os resultados apresentados são as médias de 35 simulações com o mesmo modelo de tráfego. Para estas simulações o intervalo de confiança considerado é de $95 \%$. Neste trabalho é adotado o valor unitário para os parâmetros $\alpha_{1}$ e $\alpha_{2}$.

\section{RESUltados dAS SIMUlaÇÕES DO IC_AOMDV}

Nesta seção são apresentados os resultados das simulações. Inicialmente, como prova de conceito, verifica-se a correta construção de rotas por parte do protocolo no cenário de simulação considerado. A Tabela IV apresenta a razão entre o número de rotas formadas na área mais densa e o total de rotas, para os protocolos IC_AOMDV e AOMDV, considerando a variação de velocidade e raio de transmissão dos veículos. Nota-se que o IC_AOMDV apresentou uma razão superior a $78 \%$, significando que ele constrói a maioria das rotas na região mais densa do cenário (estabelecendo, assim, rotas mais conectadas e estáveis em relação ao protocolo AOMDV).

TABELA IV

RAZÃO DE ROTAS VS. VELOCIDADE E RAIO DE TRANSMISSÃO.

\begin{tabular}{|c|c|c|}
\hline Velocidade (km/h) & IC_AOMDV (\%) & AOMDV (\%) \\
\hline $\mathbf{1 0}$ & 87,185 & 31,801 \\
\hline $\mathbf{3 0}$ & 87,422 & 30,727 \\
\hline $\mathbf{6 0}$ & 87,245 & 31,721 \\
\hline
\end{tabular}

\begin{tabular}{|c|c|c|}
\hline Raio (m) & IC_AOMDV (\%) & AOMDV (\%) \\
\hline $\mathbf{2 0 0}$ & 87,745 & 26,138 \\
\hline $\mathbf{2 5 0}$ & 81,505 & 26,661 \\
\hline $\mathbf{3 0 0}$ & 78,345 & 27,746 \\
\hline
\end{tabular}

\section{A. Análise da variação da velocidade}

Nesse caso os veículos possuem velocidades que variam entre 10 e $60 \mathrm{~km} / \mathrm{h}$ e o raio de transmissão é de $150 \mathrm{~m}$. As Figuras 3 e 4 mostram os resultados para a Taxa de Duração de Rota (TDR) e o Índice de Quebra de Rota (IQR) versus a variação da velocidade dos veículos. É possível notar que no IC_AOMDV, a TDR é maior em relação ao protocolo clássico. Isso acontece porque a métrica de decisão de rotas do IC_AOMDV utiliza as informações dos fatores influentes para ordenar as rotas. Esta métrica prioriza as rotas construídas em regiões mais densas e com mais nós favoráveis. Com isso, os enlaces são mais estáveis e não se rompem com facilidade, reduzindo o IQR. No AOMDV, onde as rotas são escolhidas apenas considerando o menor número de saltos, a probabilidade de quebra de rotas é maior, reduzindo a TDR e aumentando o IQR. A Figura 5 apresenta a Taxa de Entrega de Pacote (TEP) em função da variação da velocidade. Notase que o protocolo IC_AOMDV possui uma TEP superior ao AOMDV, pois apresenta rotas mais conectadas (com menos quebras) e assim consegue entregar mais pacotes quando comparado ao protocolo clássico.

\section{B. Análise da variação do raio de transmissão}

Neste modelo os veículos possuem velocidade máxima de $30 \mathrm{~km} / \mathrm{h}$ e o raio de transmissão varia entre 150 e $300 \mathrm{~m}$. As Figuras 6 e 7 apresentam os resultados para a Taxa de

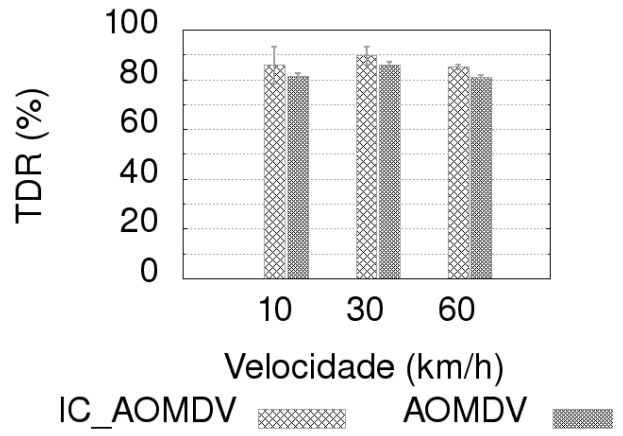

Fig. 3. TDR em função da velocidade dos nós.

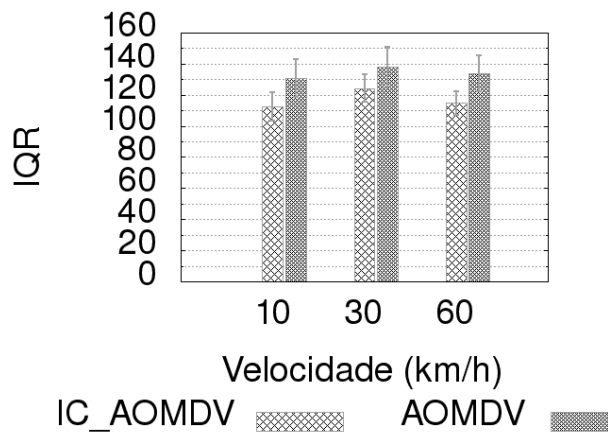

Fig. 4. IQR em função da velocidade dos nós.

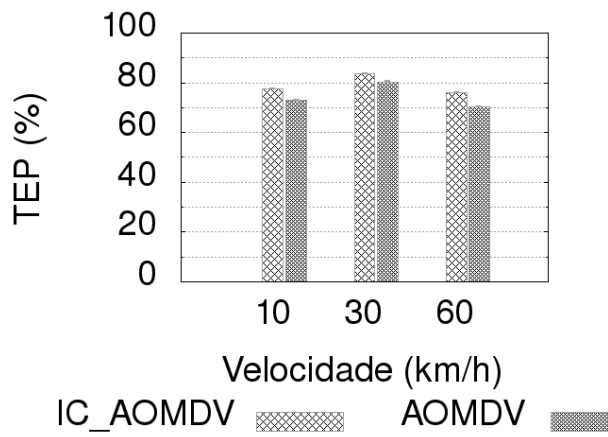

Fig. 5. TEP em função da velocidade dos nós.

Duração de Rota (TDR) e o Índice de Quebra de Rota (IQR) versus a variação do raio de transmissão dos veículos. Nota-se que a TDR do IC_AOMDV é maior em relação ao protocolo AOMDV. Embora o aumento do raio de transmissão eleve a probabilidade da formação das rotas na região menos densa e reduza o número de saltos, o IC_AOMDV é capaz de construir grande parte das rotas na região densa e com um número maior de nós favoráveis. Com isso, as rotas são mais estáveis e não se rompem com facilidade, apresentando um IQR menor quando comparado ao protocolo clássico. A Figura 8 mostra o resultado da Taxa de Entrega de Pacote (TEP) em função da variação do raio de transmissão dos veículos. Nota-se que por optar por rotas mais conectadas, o IC_AOMDV apresenta uma TEP superior ao protocolo AOMDV.

\section{Conclus Ão}

Nas VANETs, os principais desafios da comunicação estão relacionados a falta de conectividade entre os veículos devido ao reduzido tempo de duração das rotas. Uma das razões para 


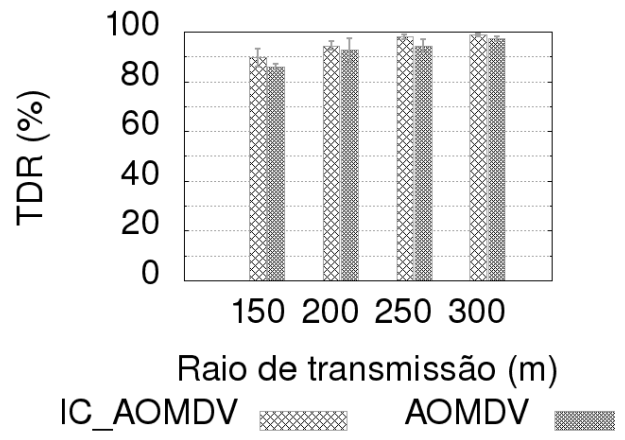

Fig. 6. TDR em função do raio de transmissão.

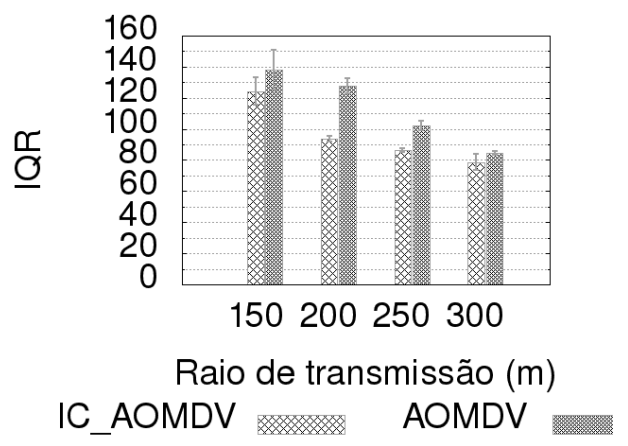

Fig. 7. IQR em função do raio de transmissão.

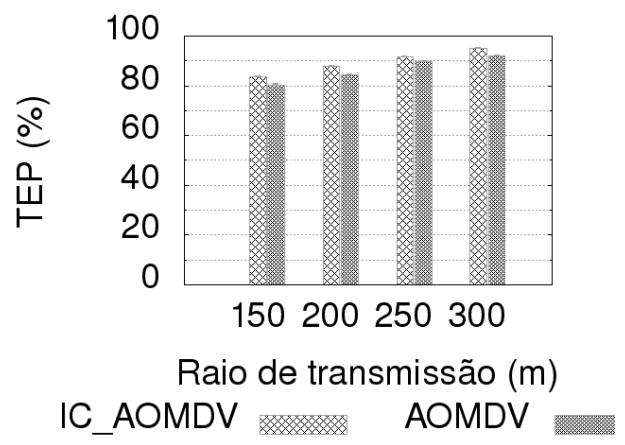

Fig. 8. TEP em função do raio de transmissão.

este tempo reduzido, é o fato dos protocolos de roteamento clássicos não se preocuparem em selecionar rotas conectadas. Por esta razão, esse trabalho desenvolveu o IC_AOMDV, um novo protocolo roteamento capaz de melhorar a conectividade e o desempenho das VANETs selecionando rotas com a máxima conectividade possível. O IC_AOMDV é uma extensão do protocolo AOMDV e foi desenvolvido para considerar os fatores influentes densidade e sentido dos deslocamentos dos veículos no momento do processo de descoberta de rotas, e assim selecionar caminhos com elevado grau de conectividade. As simulações demonstram que o IC_AOMDV produziu melhoras em todas as métricas de desempenho analisadas. Como trabalhos futuros pretende-se analisar o impacto dos parâmetros $\alpha$ no desempenho do protocolo e realizar a adaptação de outros protocolos de roteamento para que considerem os fatores influentes.

\section{APÊNDICE}

A. Determinação do sentido dos deslocamentos no NS-2

Para efeitos de simulação, foi realizada a implantação de uma lógica semelhante ao funcionamento de uma bússola digital para determinar o sentido dos deslocamento dos veículos. Para tal, o simulador de rede (NS-2) fornece nativamente, a cada $1 \mathrm{~s}$, as posições cartesianas $(X, Y)$ do nó móvel em relação a topologia [18]. Desta forma, são obtidas as posições $(x 1, y 1)$ anterior e atual $(x 2, y 2)$ do nó correspondente. Assim, é possível determinar a direção de deslocamento, mediante ao cálculo do ângulo de inclinação da reta $(\theta)$ que é formada pela diferenças entre as posições $(x, y)$, de acordo com: $\theta=\arctan (\Delta Y / \Delta X) /(180 / \pi)$, onde $\Delta X$ é a diferença das posições $(x 1, x 2)$ e $\Delta Y$ é a diferença das posições $(y 1, y 2)$. O retorno da função $\arctan (x)$ é em radianos. Entretanto, uma bussola apresenta o ângulo de deslocamento em graus, por isso é necessário converter o retorno de radianos para graus, dividindo-o por $180 / \pi$ e o resultado é expresso por valores entre $]-\pi / 2, \pi / 2[$. Como o intervalo da bússola corresponde a valores positivos entre $0^{\circ} \mathrm{e}$ $360^{\circ}$, os ângulos negativos devem ser subtraídos de $360^{\circ}$ para situá-lo entre o intervalo determinado pela bússola. Assim é possível obter o ângulo de deslocamento do nó, baseado nas suas posições atual e anterior de deslocamento.

\section{REFERENCIAS}

[1] J. Alves Junior e E. C. G. Wille, "P-AOMDV: An improved routing, protocol for $\mathrm{V} 2 \mathrm{~V}$ communication based on public transport backbones," Transactions on Emerging Telecommunications Technologies, 2016.

[2] H. Gao, C. Liu, e Y. L. X. Yang, "V2VR: Reliable Hybrid-NetworkOriented V2V Data Transmission and Routing Considering RSUs and Connectivity Probability," IEEE, 2020.

[3] J. Alves Junior e E. C. G. Wille, "Improving VANETs connectivity with a totally Ad Hoc living mobile backbone," Journal of Computer Networks e Communications, 2015.

[4] B. Sharma, M. Sharma, e R. Tomar, "A survey: Issues and challenges of vehicular Ad Hoc networks (VANETs)," SSRN Electronic Journal, 2019.

[5] J. Alves Junior e E. Wille, "P-DSDV: um protocolo de roteamento eficiente para um backbone Ad Hoc móvel," in XXXVI Simp. brasileiro de Telecomunicações e Processamento de Sinais. SBRT2018, 2018.

[6] D. de Assis; J. Alves Junior e Emilio C. G. Wille, "Análise do Tempo de Duração de Rotas em Redes Veiculares Considerando Fatores Influentes," in Anais do IV Workshop de Computação Urbana. SBC, 2020.

[7] S. Aliesawi, F. Mubarek, K. M. Ali Alheeti, e N. M. Alfahad, "UrbanAODV: An improved AODV protocol for vehicular Ad-Hoc networks in urban environment," IJET (UAE), 2018.

[8] B. M. Pamar e K. G. Maradia, "GPS-Aided AODV Routing Protocol for Manet," in Optical and Wireless Technologies, V. Janyani, M. Tiwari, G. Singh, e P. Minzioni, Eds. Springer, 2018.

[9] V. K. Muhammed Ajeer, P. C. Neelakantan, e A. V. Babu, "Network connectivity of one-dimensional Vehicular Ad Hoc Network," in 2011 Int. Conf. on Communications and Signal Processing. IEEE, 2011.

[10] R. Raw, S. Kumar Soni, N. Singh, e O. Kaiwartya, "A Probabilistic Analysis of Path Duration Using Routing Protocol in VANETs," International Journal of Vehicular Technology, 2014.

[11] R. Nazar e H. Alsabbagh, "Analysis of Path Duration in VANETs Using B-MFR Forwarding Method," IJMEC, 2016.

[12] M. Mahesh e S. Das, "Ad Hoc On-demand Multipath Distance vector routing," Wireless Communications and Mobile Computing, 2006.

[13] D. de Assis, "Técnicas para aumentar a conectividade e o desempenho das VANETs: análise do tempo de duração das rotas e um novo protocolo de roteamento," Dissertação de Mestrado, UTFPR, 2020.

[14] J. Lenz e A. Edelstein, "Magnetic Sensors and Their Applications," Sensors Journal, IEEE, 2006.

[15] NS2. (2019) The Network Simulator. [Online]. Available: https://www.isi.edu/nsnam/ns/

[16] VANETMOBISIM. (2019) Vanetmobisim. [Online]. Available: http: / /vanet . eurecom. fr

[17] D. Jiang e L. Delgrossi, "IEEE 802.11p: Towards an International Standard for Wireless Access in Vehicular Environments." in VTC Spring. IEEE, 2008.

[18] I. Teerawat e E. Hossain, Introduction to Network Simulator NS2, 2nd ed., Springer, Ed. Springer Publishing Company, Incorporated, 2018. 